\title{
Letter
}

\section{BR30 Reflector Compact Fluorescent Lamp with Improved Run-up Performance}

\author{
Xiangfen FENG \\ Research APAC, OSRAM CHINA LIGHTING LTD. 1F, Building B, No. 500 Dalian Road, Shanghai, P.R. China, 200082
}

Received December 13, 2011, Accepted January 25, 2012

\begin{abstract}
The run up characteristics of BR30 reflector compact fluorescent lamps are investigated to optimize lamp design for performance improvements. The design is optimized based on the current reflector amalgam lamp, characterized in that the solid mercury is added into the discharge tube and the paraffin wax is put at the bottom of the reflector to connect the discharge tube and the reflector bottom together. The experimental results show that the run-up time can be shortened by about $20 \mathrm{~s}$, the light efficacy can be increased by about $5 \mathrm{Im} / \mathrm{W}$, and the bottom temperature of the discharge tube can be reduced by about $25^{\circ} \mathrm{C}$ by the optimized reflector lamp design.
\end{abstract}

KEYWORDS: reflector compact fluorescent lamp, run-up time, light output, light efficacy

\section{Introduction}

Low pressure mercury discharge lamps, such as compact fluorescent lamps, rely upon mercury vapour for producing discharge. The light efficacy of such lamps is a function of the mercury vapour pressure, which is determined by the coldest part in the gas discharge tube. But when the discharge tube is located in a reflector or cover, the cold spot temperature is far beyond its optimized temperature due to the reduced heat dissipation of the reflector/cover. In order to achieve maximum light efficacy, amalgam instead of mercury is used for reflector/cover lamps, attributed to the advantage of amalgam having a wide range of optimized temperatures ${ }^{122}$. Usually the amalgam is put at the end of the discharge tube to get stable light output. But the run-up performance is poor for amalgam lamps. When the lamp is switched off, mercury is absorbed by the amalgam. This reduces luminous flux at the lamp start up because mercury vapour pressure at lamp start up is relatively low as compared with a lamp in which mercury is enclosed ${ }^{344}$.

Some attempts have been made to improve run-up performance of reflector/cover amalgam lamps by some special methods ${ }^{5)-8}$. But these methods and designs couldn It effectively improve the run-up performance or the technique is difficult to realize in practical mass production. So how to improve run-up performance is very important in designing reflector/cover compact fluorescent lamps.

A design with improved run-up characteristics for reflector/cover compact fluorescent lamps is reported in this paper. The design is optimized based on the current reflector amalgam lamp, characterized in that the solid mercury is added into the discharge tube to shorten the run-up time and the paraffin wax is used to connect the discharge tube and the reflector bottom together to improve light output and light efficacy.

\section{Experiments}

Typical BR30 reflector lamps are used for the experiment. The spiral discharge tube is installed in a BR30 reflector. The spiral tube and the reflector combined with the ballast, the base and the socket are put together as a whole lamp system with the input power of $16 \mathrm{~W}$, as shown in Figure 1(a). The amalgam is put at the end of one of the exhaust tube and is supported by a glass rod thus it will not drop into the discharge tube. The amalgam is composed by $\mathrm{Bi} / \mathrm{In} / \mathrm{Hg}$ alloy with $\mathrm{Hg}$ composition of $4.5 \%$. In order to quicken light output at the lamp starting up process, the indium flag as auxiliary amalgam is welded on the metal conductive wire inside the tube, and the metal wire outside the tube is connected with the ballast to provide power to the electrodes to produce electrons to induce discharge inside the tube. The indium content on the flag is $0.2 \mathrm{mg}$ and the flag dimension is $2 \mathrm{~mm} \times 5 \mathrm{~mm}$. The distance between the flag and the electrode is $2.5 \mathrm{~mm}$. The spiral discharge tube is double spiral structure and the two spirals are connected at the bottom as a whole discharge system. The outer diameter of the tube and the spiral is respectively $9.5 \mathrm{~mm}$ and $45.8 \mathrm{~mm}$. Each spiral has 1.75 circles and the centre distance of the single circle is $22 \mathrm{~mm}$.

To improve the performance of this reflector lamp, the design is improved based on the current design by adding the solid mercury into the discharge tube, which 


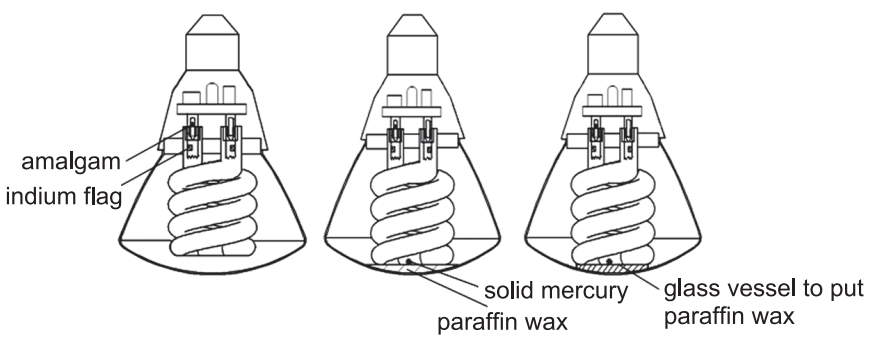

(a)

(b)

(c)

Figure 1 Several designs for BR30 compact fluorescent lamp

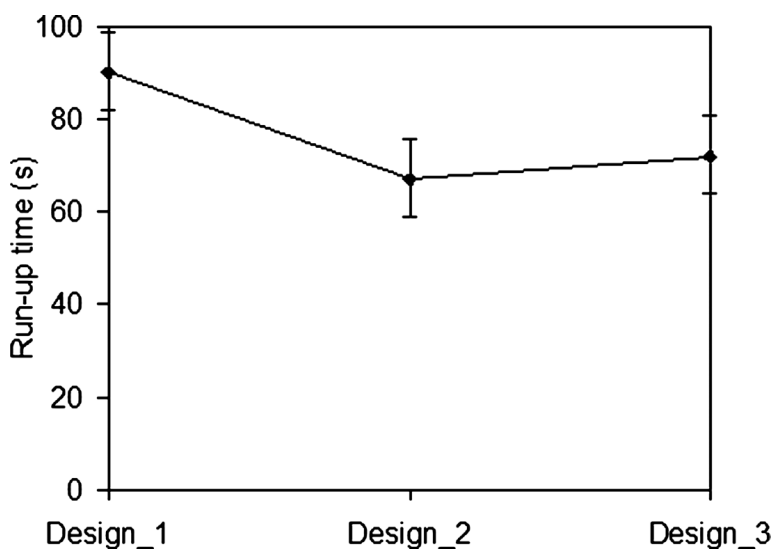

Figure 2 Run up time for three designs

is located at the tube bottom when the lamp is operated in the base-up position. The paraffin wax is put at the reflector bottom to connect the tube and the reflector bottom together. The reported design is shown in Figure 1(b). All lamps have been operated for $100 \mathrm{~h}$ in the base-up position in an open-air rack. The test data are averaged for five lamps for every group of experiments.

A photometry measurement system (Everfine) is used for electrical and photometry performance measurements, which includes an integrating sphere of $1 \mathrm{~m}$ diameter, a high accuracy array spectrometer (HAAS2000) scanning over the wavelength from $380 \mathrm{~nm}$ to $780 \mathrm{~nm}$ with $0.1 \mathrm{~nm}$ resolution, an AC power supply (TPS500B), and a power meter (PF9800). Run up time (time to reach $80 \%$ stable lumen) is measured in the integrating sphere after $24 \mathrm{~h}$ switched off in $25^{\circ} \mathrm{C}$ environment. The temperature is measured by a thermocouple (Fluke, $51 \mathrm{P}$ ) through a thermocouple being directly connected on the testing point in the lamp.

\section{Results}

The run-up time, the photometry performance, and the temperature are compared respectively for the design of (1) the current lamp design, (2) the solid mercury added in the discharge tube, (3) the solid mercury added in the discharge tube and the discharge tube and the reflector bottom are connected by the paraffin wax.

Figure 2 shows the run-up time for three designs. It

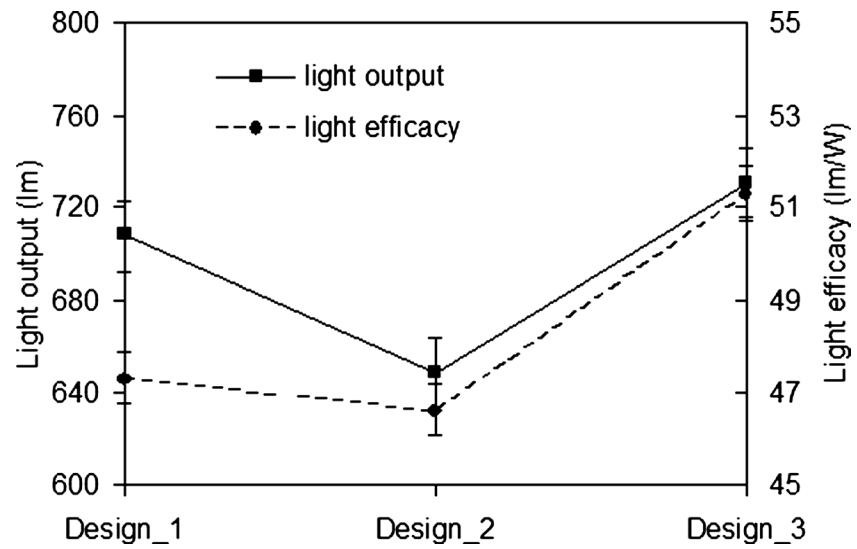

Figure 3 Light output and light efficacy for three designs

can be seen that when the solid mercury is added into the discharge tube besides the main amalgam located in the exhaust tube, the run-up time can be shortened by about $20 \mathrm{~s}$ compared with its current design. When there is only amalgam and the indium flag located at two ends, it needs to take long time for the mercury gas atoms to diffuse from the two ends to the tube bottom along the long narrow discharge path thus resulting in slow light diffusion. The most effective method for speeding the rising of the luminous flux at lamp start up is to design to keep some mercury left in the discharge tube when the lamp is turned off. Therefore, when the solid mercury is added at the bottom of the discharge tube, more mercury will be excited and ionized so some light can also be produced from the middle of the tube and the light diffusion distance can be shortened. Thus, the time it takes for the light filling in the whole discharge tube is shortened. The light rising speed is increased by the mercury left inside the discharge tube, which helps to produce discharge when the lamp is ignited.

Figure 3 shows the light output and the light efficacy for the three designs. Although the run up time is shortened when the solid mercury is added, the stable light output is reduced by about $10 \%$ due to the mercury pressure in the tube tip being beyond its optimized pressure. In the case of that the tube bottom and the reflector bottom are connected by the paraffin wax, the light output can be improved again and the light efficacy can be increased by about $5 \mathrm{~lm} / \mathrm{W}$ while at the same time the run-up time is still shortened.

The disadvantage of adding mercury into the discharge tube is that the light output is decreased. In order to improve light output, the paraffin wax is used to connect the discharge tube and the reflector bottom so to reduce the tube bottom temperature. When the lamp is operating, the paraffin wax will absorb heat from the discharge tube and will melt. The melted paraffin wax conducts heat from the burner to the reflector. There- 


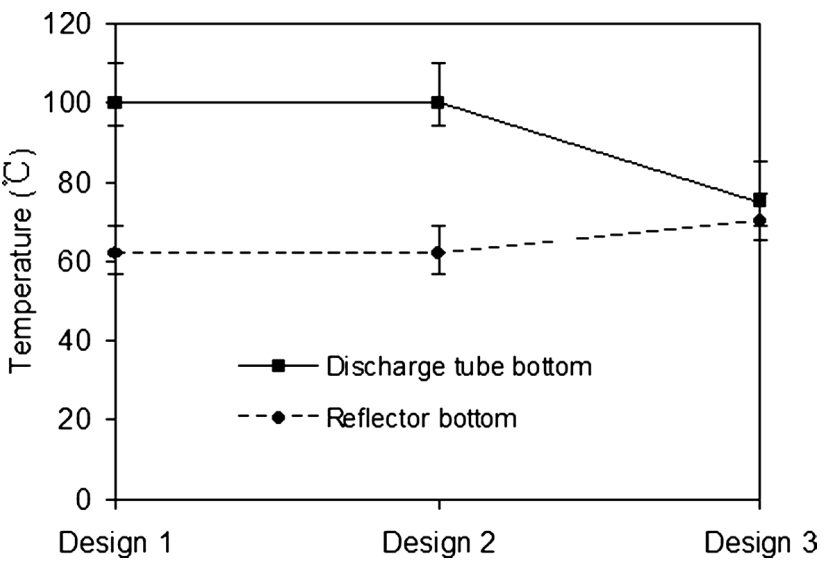

Figure 4 Temperature of the discharge tube bottom and the reflector bottom for three designs

fore, the bottom temperature of the discharge tube can be decreased by dissipating heat to the paraffin and then to the reflector, and at last to the ambient environment. When there is an air gap between the discharge tube and the reflector, the thermal radiation is the only way to dissipate heat from the discharge tube. Figure 4 shows the bottom temperature respectively of the discharge tube and the reflector for three designs. It can be seen that when there is paraffin wax at the bottom of the discharge tube, the tube bottom temperature can be reduced by about $25^{\circ} \mathrm{C}$ by thermal conduction thus the mercury pressure will not be beyond its optimized pressure too much, and therefore the light output and the light efficacy can be increased. Also the melted paraffin is transparent for visible light and it will not influence the light transmission from the glass cover. When the lamp is switched off, the paraffin will gradually solidify again. So the solid mercury and the paraffin wax together can help both fast light rising and high light output and efficacy.

By this design, the run-up performance for reflector/ cover amalgam lamps can be improved while at the same time high light output and high light efficacy can be achieved. This kind of design of adding the solid mercury in the discharge tube and enclosing the paraffin wax in the reflector is not limited to BR30 reflector lamps, but it is also suitable for other kinds of reflector lamps and cover lamps with amalgam.

Considering the molten paraffin is fluid when the lamp is turned on, an improved design for the covered/ reflector is proposed as shown in Figure 1c. A glass vessel connected to the reflector bottom is used to hold the paraffin. The tube bottom is tightly covered on the glass vessel to prevent the paraffin flowing.

\section{Conclusions}

The run-up performance of BR30 reflector compact fluorescent lamp is improved while at the same time increased light output and light efficacy are achieved by the reported lamp design. The following advantages are obtained by the technical features disclosed in this paper:

(1) The solid mercury added in the discharge tube can increase the luminous flux rising at lamp start up thus to shorten run-up time and increase run-up performance.

(2) The paraffin wax can absorb heat from the discharge tube to melt and conduct heat to the reflector and then to the ambient air so to decrease the cold spot temperature of the discharge tube effectively.

(3) The light output and light efficacy can be increased by the low cold temperature of the discharge tube thus the mercury pressure inside the discharge tube is close to its optimized pressure.

(4) The melted paraffin wax has good visible light transmissivity thus it doesn It reduce light output from the glass cover.

\section{References}

(1) Janke D., Madarász J., Lukács S., et al.: DSC Investigation of Amalgam Formation in Bi-Sn-Hg System Doped with Indium, Journal of Thermal Analysis and Calorimetry, 96-2, pp. 443-447 (2009).

(2) Lankhorst M. H. R., Keur W. and van Hal H. A. M.: Amalgams for Fluorescent Lamps Part II: The Systems $\mathrm{Bi}-\mathrm{Pb}-\mathrm{Hg}$ and $\mathrm{Bi}-\mathrm{Pb}-\mathrm{Au}-\mathrm{Hg}$, Journal of Alloys and Compounds, 309, pp. 188-196 (2000).

(3) Yasuda T. and Kando M.: Improvement of the Luminous Run-up Characteristics in Ballast-integrated Compact Fluorescent Lamps Covered with Outer Globes, The Illuminating Engineering Society of North America, 4-1, pp. 57-70 (2007).

(4) Yasuda T. and Kando M.: Experimental Study on the Mercury Vapor Pressures in Amalgam-dosed Discharge Tubes for Compact Fluorescent Lamps during Switch-off Period, J. Light \& Vis. Env., 32-1, pp. 33-38 (2008).

(5) Myojo M. and Namura T.: Discharge Lamp Having an Amalgam within a Barrier Means, US Patent 5828169 (1998).

(6) Johannes W., Bogert V. D., Hermannus H., et al: Low Pressure Mercury Vapor Discharge Lamp with Improved Auxiliary Amalgam, US Patent 6707246 B1 (2004).

(7) Beverly C. J. B., Menelly R. A. and Mass D.: Electric Discharge Lamp with Integrated Pressure Regulator, US Patent 3227907 (1966).

(8) Menelly R. A. and Mass D.: Fluorescent Electric Discharge Lamp Having the Phosphor Particles Admixed with Indium Powder Particles, US Patent 3339100 (1967). 